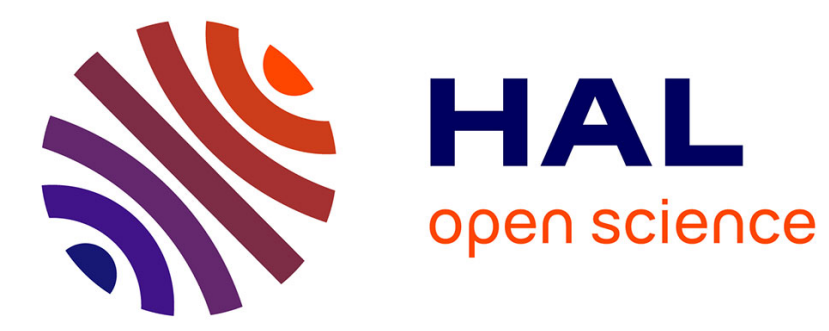

\title{
An asymptotic approach to thin film adhesion
}

Frédéric Lebon, Raffaella Rizzoni

\section{To cite this version:}

Frédéric Lebon, Raffaella Rizzoni. An asymptotic approach to thin film adhesion. Eleventh International Conference on Computational Structures Technology, Sep 2012, Dubrovnik, Croatia. 10.4203/ccp.99.144 . hal-00740766

\section{HAL Id: hal-00740766 \\ https://hal.science/hal-00740766}

Submitted on 19 Nov 2021

HAL is a multi-disciplinary open access archive for the deposit and dissemination of scientific research documents, whether they are published or not. The documents may come from teaching and research institutions in France or abroad, or from public or private research centers.
L'archive ouverte pluridisciplinaire HAL, est destinée au dépôt et à la diffusion de documents scientifiques de niveau recherche, publiés ou non, émanant des établissements d'enseignement et de recherche français ou étrangers, des laboratoires publics ou privés. 


\title{
An Asymptotic Approach to Thin Film Adhesion
}

\author{
Frédéric Lebon ${ }^{1}$ and Raffaella Rizzoni ${ }^{2}$ \\ 1 M\&S - Matériaux et Structures, LMA - Laboratoire de Mécanique et d'Acoustique, France \\ 2 Dipartimento di Ingegneria, Université de Ferrara, Italy
}

\begin{abstract}
A common approach to the modeling of very thin adhesive films is their replacement with an equivalent contact law, prescribing the jumps in the displacement and traction vector fields at the limit interface as the film thickness goes to zero. From the geometrical point of view, the adhesive film is eliminated, although it is accounted for mechanically. Recently, we analyzed several cases to obtain the equivalent contact laws: soft films [17, 18]; adhesive films governed by a non convex energy [12]; linearly elastic adhesive films having stiffness comparable with the adherents $[19,13]$; imperfect adhesion between flat adhesive films and the adherents [14]; joints with mismatch strain between the adhesive and the adherents [16]. In this paper, we extend the results obtained in $[13,14]$ to the case of a thin curvilinear elastic anisotropic adhesive undergoing plane deformations. The asymptotic method proposed in [14] and based on the energy minimization is used. After obtaining the contact law in a general system of curvilinear coordinates, we analyze the gluing between two circular adherents (figure 2), a case of significant importance for composite materials which often contain fibers or particles.
\end{abstract}

Keywords: thin film; elasticity; asymptotic analysis; curvilinear coordinates.

\section{Introduction}

Interphase regions are thin layers of a third material between the composite constituents, fibers or particles and matrix, which are due to mechanical imperfections, fiber treatments, and various modifications of the matrix in the vicinity of the fibers. The presence of interphases influences the load transfers from the 
matrix to fibers and it can have a strong effect on the overall mechanical response of the composite. The existence of interphase regions of finite but small dimensions poses also several numerical problems: in particular, the number of degrees of freedom adopted in studies using a finite element approach can be very large, which affects the convergence and the accuracy of the solution.

A classical approach is to replace the three-phase problem of two constituents and an interphase perfectly adherent to them with a two-phase problem, in which the thin interphase is substituted by a mechanically equivalent interface of vanishing thickness $\varepsilon[6,7,8,20,10,11,20,21,22,24,23,25]$. In particular, several studies were concerned with the derivation of the imperfect interface, modeling the effect of a thin interphase of known elastic properties [1, 2, 3, 9, 12, 21, 25]. The method of the derivation can be based on different mathematical techniques: gamma-convergence, variational analysis, asymptotic expansions and numerical studies [15]. Using these methods, it has been established that at the first order $(\varepsilon \rightarrow 0)$ flat stiff elastic interphases are equivalent to perfect interfaces, at which the stress and the displacement vectors are continuous. At a higher order (the second term in the expansion), an imperfect interface model is obtained, with a contact condition involving the first order displacement and traction vectors and their derivatives $[1,13,14,19]$. The higher order term, giving rise to the imperfect interface model, can be interpreted as a correction of the leading solution corresponding to the perfect interface model.

In this paper, we extend the previous results to the case of a curved thin adhesive made of linear elastic anistropic material and undergoing plane deformations. Section 2 deals with the equilibrium problem of the interphase/adherents system. The asymptotic analysis is introduced in Section 3, where the existence of expansions in series of the displacements and stress vector fields in terms of the small parameter describing the thickness is assumed. Another assumption is that we can obtain the fields which are stationary points of the energy of the system by finding the stationary points of the energies obtained at each level in the expansion. This method provides two types of relations: either an interface relation or an equilibrium relation. In particular, at the order -1 we obtain the continuity of the displacement vector field at order zero; at the order 1 we obtain the continuity of the traction vector field at order zero and relation (54), which determines the jump at the interface on the displacement vector field at order one; at the order 2 we find relation (64), which prescribes the jump of traction at order one in terms of the displacement and traction vector fields at order zero. Relations $(54,64)$ are associated with an imperfect interface law, non-local in the sense that it involves tangential derivatives of the fields at order zero.

In Section 4 we specialize the imperfect interface law, given by relations (54, 64) and obtained in a general system of curvilinear coordinates, to the case of a circular inclusion bounded by a thin isotropic adhesive layer. 


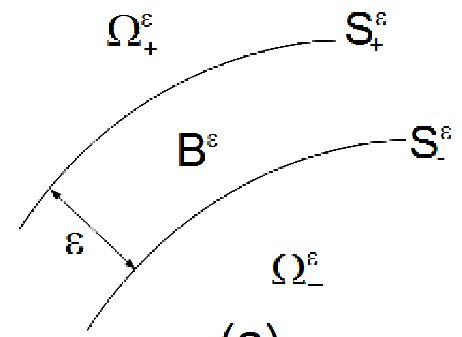

(a)

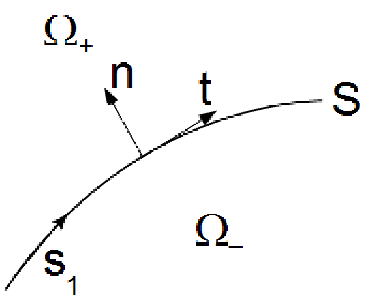

(b)

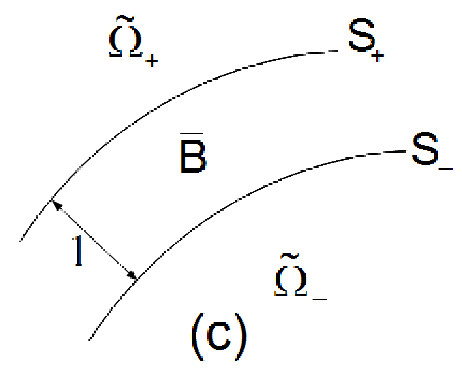

Figure 1: (a) Initial configuration with a thin interphase $B^{\varepsilon}$ of small thickness $\varepsilon$ placed between two adherents; (b) limit configuration, where the interphase $B^{\varepsilon}$ is replaced by the interface $S$; (c) rescaled configuration with an interphase $\bar{B}$ of unit thickness placed between the adherents.

\section{Statement of the problem}

Consider a two-dimensional plane strain configuration in which a thin elastic adhesive interphase $B^{\varepsilon} \in \mathbb{R}^{2}$ of constant thickness $\varepsilon$ separates two elastic adherents $\Omega^{\varepsilon}+, \Omega^{\varepsilon}-\in \mathbb{R}^{2}$, see figure 1 . The two interfaces between the three region $\Omega^{\varepsilon}{ }_{+}, \Omega^{\varepsilon}$ and $B^{\varepsilon}$ will be denoted $S^{\varepsilon}+$ and $S^{\varepsilon}$. These interfaces are assumed to be perfect with the usual assumption that the displacement vector, $u^{\varepsilon}$, and the stress vector, $\sigma^{\varepsilon} n$, are continuous on $S^{\varepsilon}{ }_{ \pm}$. It is the purpose here to substitute the thin interphase $B^{\varepsilon}$ by a curve $S$ separating the adherents $\Omega^{\varepsilon}{ }_{+}, \Omega^{\varepsilon}$, and to determine the interface conditions on the displacement and tractions across $S$ equivalent to the three phase configuration with perfect interface conditions.

The three regions are anisotropic, homogeneous and linear elastic and let $\mathbf{a}_{ \pm}$ denote the elasticity tensors of the regions $\Omega^{\varepsilon}{ }_{ \pm}$, and $\mathbf{b}$ the elasticity tensor of the interphase $B^{\varepsilon}$. Denoted $e$ the strain tensor

$$
e\left(u^{\varepsilon}\right)=\frac{1}{2}\left(\nabla u^{\varepsilon}+\left(\nabla u^{\varepsilon}\right)^{T}\right)
$$

linear elasticity gives the Cauchy stress tensor $\sigma^{\varepsilon}$ as follows:

$$
\begin{aligned}
\sigma^{\varepsilon} & =\mathbf{b}(e) \text { in } B^{\varepsilon}, \\
\sigma^{\varepsilon} & =\mathbf{a}_{ \pm}(e) \text { in }{\Omega^{\varepsilon}}^{\varepsilon} .
\end{aligned}
$$


Let us introduce a rectangular system of Cartesian coordinates $O x_{1} x_{2} x_{3}$ so that the plane $x_{3}=0$ coincides with the plane of the interphase. The elasticity tensors are assumed to satisfy the following assumptions:

$$
\left\{\begin{array}{l}
\mathbf{a}_{ \pm}, \mathbf{b} \in L^{\infty}\left(\Omega^{\varepsilon}+\cup \Omega^{\varepsilon}\right) \\
\mathbf{a}_{ \pm i j k l}=\mathbf{a}_{ \pm k l i j}=\mathbf{a}_{ \pm j i l k}=\mathbf{a}_{ \pm i j l k} \\
\mathbf{b}_{i j k l}=\mathbf{b}_{k l i j}=\mathbf{b}_{j i l k}=\mathbf{b}_{i j l k} \\
\exists \eta_{ \pm}, \eta>0: \mathbf{a}_{ \pm}(e) \cdot(e) \geq \eta_{ \pm}|e|^{2} \\
\mathbf{b}(e) \cdot(e) \geq \eta|e|^{2}, \quad \forall e: e=e^{T} .
\end{array}\right.
$$

A body force density $f \in\left(L^{2}\left(\Omega^{\varepsilon}{ }_{+} \cup \Omega^{\varepsilon}{ }_{-}\right)\right)^{3}$ is assumed to be applied to $\Omega^{\varepsilon}+\cup \Omega^{\varepsilon}{ }_{-}$ and a surface force density $g \in\left(L^{2}\left(\Gamma_{g}\right)\right)^{3}$ to $\Gamma_{g} \subset \partial \Omega^{\varepsilon}+\cup \partial \Omega^{\varepsilon}{ }_{-}$. On $\Gamma_{u}=$ $\left(\partial \Omega^{\varepsilon}+\cup \partial \Omega_{-}^{\varepsilon}\right) \backslash \Gamma_{g}$, homogeneous boundary conditions are prescribed

$$
u^{\varepsilon}=0 \quad \text { on } \Gamma_{u} \text {. }
$$

The equilibrium configurations of the three-phase composite body are the minimizers of the total energy

$$
\begin{aligned}
E^{\varepsilon}(u)= & \int_{\Omega^{\varepsilon} \pm}\left(\frac{1}{2} \mathbf{a}_{ \pm}(e(u)) \cdot e(u)-f \cdot u\right) d x-\int_{\Gamma_{g}} g \cdot u d s_{x} \\
& +\int_{B^{\varepsilon}} \frac{1}{2} \mathbf{b}(e(u)) \cdot e(u) d x .
\end{aligned}
$$

in the space of kinematically admissible displacements

$$
V^{\varepsilon}=\left\{u \in H\left(\Omega^{\varepsilon} ; R^{3}\right): u=0 \text { on } \Gamma_{u}\right\}
$$

where $H\left(\Omega^{\varepsilon} ; R^{3}\right)$ is the space of the vector-valued functions on the set $\Omega^{\varepsilon}:=$ $\Omega^{\varepsilon}+\cup \Omega^{\varepsilon}-\cup B^{\varepsilon} \cup S^{\varepsilon}{ }_{+} \cup S^{\varepsilon}{ }_{-}$, which are continuous and differentiable as many times as necessary. In view of the above regularity assumptions on $\mathbf{a}_{ \pm}, \mathbf{b}, f, g$, the existence of a unique minimizer $u^{\varepsilon}$ in $V^{\varepsilon}$ is ensured [5, Theorem 6.3-2].

We assume that a parametric representation of $S$ has been introduced in $\mathbb{R}^{2}$, $x=\phi\left(s_{1}\right), s_{1} \in(a, b)$. We also assume that the curves $S^{\varepsilon}+$ and $S^{\varepsilon}$ - are parallel to one another and we introduce the following representation for $B^{\varepsilon}$ :

$$
B^{\varepsilon}=\left\{x \in \mathbb{R}^{2}: x=\phi\left(s_{1}\right)+s_{2} n\left(s_{1}\right), \quad\left(s_{1}, s_{2}\right) \in(a, b) \times(-\varepsilon / 2, \varepsilon / 2)\right\},
$$

where $n$ is the unit normal vector to $S$ pointing from $\Omega^{\varepsilon}{ }_{-}$to $\Omega^{\varepsilon}+$. It is convenient to work with the system of planar orthogonal curvilinear coordinates $s_{1}, s_{2}$. Let $h^{\varepsilon}{ }_{1}, h^{\varepsilon}{ }_{2}$ denote the metric coefficient of the curvilinear coordinated system, and for the present parallel system we have

$$
\begin{aligned}
& h_{1}^{\varepsilon_{1}}=\sqrt{x_{, 1} \cdot x_{, 1}}=\sqrt{\phi_{, 1}+s_{2} n_{, 1}}, \\
& h_{2}^{\varepsilon_{2}}=\sqrt{x_{, 2} \cdot x_{, 2}}=1 .
\end{aligned}
$$


In the curvilinear system, the displacement gradient takes the form:

$$
\nabla u=\left(U_{1}\left|U_{2}\right| 0\right)
$$

where the notation $(a|b| c)$ indicates the matrix whose columns are the vectors $a, b, c$, and the vectors $U_{i}, i=1,2$, are defined as follows:

$$
\begin{aligned}
U_{1} & :=\frac{u_{, 1}}{h^{\varepsilon}}+\frac{h^{\varepsilon} 1,2}{h^{\varepsilon} 1} v, \\
U_{2} & :=u_{, 2} \\
v & :=u \wedge e_{3} .
\end{aligned}
$$

In $(12,13)$ a comma is used to denote partial differentiation, and $e_{3}$ is the unit vector normal to the plane of the interphase. In curvilinear coordinates, we have

$$
\begin{aligned}
E^{\varepsilon}(u)= & \int_{D^{\varepsilon} \pm}\left(\frac{1}{2} \mathbf{a}_{ \pm}(e(u)) \cdot e(u)-f \cdot u\right) h_{1}^{\varepsilon} d s-\int_{I_{g}} g \cdot u h_{s}^{\varepsilon} d \alpha \\
& +\int_{D^{\varepsilon}} \frac{1}{2}(\mathbf{B}(U) \cdot U) h_{1}^{\varepsilon} d s
\end{aligned}
$$

where $D^{\varepsilon}:=(a, b) \times(-\varepsilon / 2, \varepsilon / 2), D^{\varepsilon}{ }_{ \pm}$are the new domains of integration corresponding to $\Omega^{\varepsilon}$, after the change of variables from the Cartesian coordinates to the curvilinear ones, and $h_{1}^{\varepsilon}$ is the Jacobian of the transformation; $\alpha \in I_{g}:=\left(\alpha_{1}, \alpha_{2}\right)$ is the parameter of the parametric representation chosen for $\Gamma_{g}$, and $h_{s}^{\varepsilon}$ is the Jacobian of the surface transformation; $U:=\left(U_{1}, U_{2}\right)$ is a $2 \times 1$ block matrix; $\mathbf{B}$ is a $2 \times 2$ block matrix whose blocks, $B^{\alpha \beta}, \alpha, \beta=1,2$, are the matrices with components defined by the relations

$$
B_{i j}^{\alpha \beta}:=\mathbf{b}_{i \alpha j \beta} .
$$

In view of the symmetry properties of the elasticity tensor $\mathbf{b}$, the matrices $B^{\alpha \beta}$ have the property that $B^{\beta \alpha}=\left(B^{\alpha \beta}\right)^{T}, \alpha, \beta=1,2$. Therefore, $\mathbf{B}$ is symmetric.

\section{Asymptotic analysis}

In the following, we sketch the asymptotic method based on the energy minimization which was introduced in [14]. The first step of the method is a change of variables, in order to reformulate the equilibrium problem in an interphase domain independent of $\varepsilon$ :

$$
\begin{aligned}
\left(t_{1}, t_{2}\right) & =\bar{p}\left(s_{1}, s_{2}\right):=\left(s_{1}, s_{2} \varepsilon^{-1}\right), \quad\left(s_{1}, s_{2}\right) \in(a, b) \times(-\varepsilon / 2, \varepsilon / 2), \\
z & =\tilde{p}(x):=x \pm\left(\frac{\varepsilon}{2} \mp \frac{1}{2}\right) n, \quad x \in \Omega^{\varepsilon}{ }_{ \pm} .
\end{aligned}
$$

After the change of variables, the interphase occupies the domain

$$
\bar{B}=\left\{x \in \mathbb{R}^{2}: x=\phi\left(t_{1}\right)+t_{2} n\left(t_{1}\right), \quad\left(t_{1}, t_{2}\right) \in(a, b) \times(-1 / 2,1 / 2)\right\},
$$


and the adherents occupy the domains $\tilde{\Omega}_{ \pm}=\Omega^{\varepsilon}{ }_{ \pm} \pm 1 / 2(1-\varepsilon) n$. Let $S_{ \pm}$denote the interfaces between the interphase and the two bodies after rescaling, and let $\Omega=\tilde{\Omega}_{+} \cup \tilde{\Omega}_{-} \cup \bar{B} \cup S_{+} \cup S_{-}$denote the configuration of the composite body after the change of variables. Lastly, let $\tilde{\Gamma_{u}}$ and $\tilde{\Gamma_{g}}$ denote the shifts of $\Gamma_{u}$ and $\Gamma_{g}$, respectively.

Let $\tilde{u}_{ \pm}^{\varepsilon}=u^{\varepsilon} \circ \tilde{p}^{-1}$ be the displacement from the adherents $\Omega_{ \pm}$adjacent to the rescaled interphase and let $\bar{u}^{\varepsilon}=u^{\varepsilon} \circ \bar{p}^{-1}$ be the displacement from the rescaled interphase. In view of the condition of perfect interfaces between the adherents and the rescaled interphase, we have that $\tilde{u}_{ \pm}^{\varepsilon}(z)=\bar{u}^{\varepsilon}(z), z \in S_{ \pm}$. Let $\tilde{f}:=f \circ \tilde{p}^{-1}$ and $\tilde{g}:=g \circ \tilde{p}^{-1}$ denote the rescaled external forces, and $\tilde{h}^{\varepsilon}:=h^{\varepsilon} \circ \tilde{p}^{-1}$, $\tilde{h}_{s}^{\varepsilon}:=h_{s}^{\varepsilon} \circ \tilde{p}^{-1}, \bar{h}^{\varepsilon}:=h^{\varepsilon} \circ \bar{p}^{-1}$, the rescaled metric coefficients.

With these assumptions and denoted with a tilde domains and fields associated with the adherents and with a bar domains and fields associated with the interphase, the rescaled energy takes the form

$$
\begin{aligned}
\mathcal{E}^{\varepsilon}\left(\tilde{u}_{ \pm}^{\varepsilon}, \bar{u}^{\varepsilon}\right):=\int_{\bar{D}_{ \pm}} & \left(\frac{1}{2} \mathbf{a}_{ \pm}\left(e\left(\tilde{u}_{ \pm}^{\varepsilon}\right)\right) \cdot e\left(\tilde{u}_{ \pm}^{\varepsilon}\right)-\tilde{f} \cdot \tilde{u}_{ \pm}^{\varepsilon}\right) \tilde{h}_{1}^{\varepsilon} d t-\int_{\tilde{I}_{g}}\left(\tilde{g} \cdot \tilde{u}_{ \pm}^{\varepsilon}\right) \tilde{h}_{s}^{\varepsilon} d \tilde{\alpha} \\
& +\int_{\bar{D}} \frac{1}{2}\left(\mathbf{B}\left(\bar{U}^{\varepsilon}\right) \cdot \bar{U}^{\varepsilon}\right) \varepsilon \bar{h}^{\varepsilon}{ }_{1} d t
\end{aligned}
$$

where $\bar{D}:=(a, b) \times(-1 / 2,+1 / 2)$, and $\bar{U}^{\varepsilon}:=\left(\bar{U}_{1}^{\varepsilon}, \bar{U}_{2}^{\varepsilon}\right)$ with

$$
\begin{aligned}
\bar{U}_{1}^{\varepsilon} & :=\frac{\bar{u}_{, 1}^{\varepsilon}}{\bar{h}^{\varepsilon}{ }_{1}}+\frac{\bar{h}^{\varepsilon} 1,2}{\varepsilon \bar{h}^{\varepsilon}} \bar{v}^{\varepsilon}, \\
\bar{U}_{2}^{\varepsilon} & :=\frac{\bar{u}_{, 2}^{\varepsilon}}{\varepsilon} .
\end{aligned}
$$

The rescaled equilibrium problem $\tilde{P}^{\varepsilon}$ can be formulated as follows: find the pair $\left(\tilde{u}_{ \pm}^{\varepsilon}, \bar{u}^{\varepsilon}\right)$ minimizing the energy (20) in the set of displacements

$$
V=\left\{\left(\tilde{u}_{ \pm}, \bar{u}\right) \in H\left(\Omega_{ \pm} ; R^{3}\right) \times H\left(\bar{B} ; R^{3}\right): \tilde{u}_{ \pm}=\bar{u} \text { on } S_{ \pm}, \tilde{u}_{ \pm}=0 \text { on } \tilde{\Gamma}_{u}\right\}
$$

The next step of the asymptotic method followed in the present paper is the expansion of the displacement vectors $\tilde{u}_{ \pm}^{\varepsilon}, \bar{u}^{\varepsilon}$ and of the (rescaled) metric coefficients in power of $\varepsilon$ :

$$
\begin{aligned}
\tilde{u}_{ \pm}^{\varepsilon} & =\tilde{u}_{ \pm}^{0}+\varepsilon \tilde{u}_{ \pm}^{1}+\varepsilon^{2} \tilde{u}_{ \pm}^{2}+o\left(\varepsilon^{2}\right) \\
\bar{u}^{\varepsilon} & =\bar{u}^{0}+\varepsilon \bar{u}^{1}+\varepsilon^{2} \bar{u}^{2}+o\left(\varepsilon^{2}\right) \\
\bar{h}^{\varepsilon} & =\bar{h}_{1}^{0}+\varepsilon \bar{h}_{1}^{1}+\varepsilon^{2} \bar{h}_{1}^{2}+o\left(\varepsilon^{2}\right) \\
\tilde{h}_{1}^{\varepsilon} & =\tilde{h}_{1}^{0}+\varepsilon \tilde{h}_{1}^{1}+\varepsilon^{2} \tilde{h}_{1}^{2}+o\left(\varepsilon^{2}\right), \\
\tilde{h}_{s}^{\varepsilon} & =\tilde{h}_{s}^{0}+\varepsilon \tilde{h}_{s}^{1}+\varepsilon^{2} \tilde{h}_{s}^{2}+o\left(\varepsilon^{2}\right) .
\end{aligned}
$$


We also have

$$
\begin{aligned}
\bar{h}_{1}^{0} & =\left|\phi_{, 1}\right|=\tilde{h}_{1}^{0} \\
\bar{h}_{1}^{1} & =t_{2}\left(\frac{\phi_{, 1} \cdot n_{, 1}}{\left|\phi_{, 1}\right|}\right) \\
\bar{h}_{1}^{1} & =t_{2}^{2}\left(\frac{\left|\phi_{, 1}\right|^{2}\left|n_{, 1}\right|^{2}-\left(\phi_{, 1} \cdot n_{, 1}\right)^{2}}{2\left|\phi_{, 1}\right|^{3}}\right) .
\end{aligned}
$$

Substituting the above expansions into the rescaled energy, we obtain

$$
\begin{aligned}
\mathcal{E}^{\varepsilon}\left(\tilde{u}_{ \pm}, \bar{u}\right)=\frac{1}{\varepsilon} \mathcal{E}^{-1}\left(\bar{u}^{0}\right)+\mathcal{E}^{0}\left(\tilde{u}_{ \pm}^{0}, \bar{u}^{0}, \bar{u}^{1}\right)+\varepsilon \mathcal{E}^{1}\left(\tilde{u}_{ \pm}^{0}, \tilde{u}_{ \pm}^{1}, \bar{u}^{0}, \bar{u}^{1}, \bar{u}^{2}\right)+ \\
+\varepsilon^{2} \mathcal{E}^{2}\left(\tilde{u}_{ \pm}^{0}, \tilde{u}_{ \pm}^{1}, \tilde{u}_{ \pm}^{2}, \bar{u}^{0}, \bar{u}^{1}, \bar{u}^{2}, \bar{u}^{3}\right)+o\left(\varepsilon^{2}\right)
\end{aligned}
$$

where

$$
\begin{aligned}
\mathcal{E}^{-1}\left(\bar{u}^{0}\right):= & \int_{\bar{D}} \frac{1}{2}\left(\left(\mathbf{B} \bar{U}^{-1}\right) \cdot \bar{U}^{-1}\right) \bar{h}_{1}^{0} d t \\
\mathcal{E}^{0}\left(\tilde{u}_{ \pm}^{0}, \bar{u}^{0}, \bar{u}^{1}\right):= & \int_{\bar{D}_{ \pm}}\left(\frac{1}{2} \mathbf{a}_{ \pm}\left(e\left(\tilde{u}_{ \pm}^{0}\right)\right) \cdot e\left(\tilde{u}_{ \pm}^{0}\right)-\tilde{f} \cdot \tilde{u}_{ \pm}^{0}\right) \tilde{h}_{1}^{0} d t-\int_{\tilde{I}_{g}}\left(\tilde{g} \cdot \tilde{u}_{ \pm}^{0}\right) \tilde{h}_{s}^{0} d \tilde{\alpha} \\
& +\int_{\bar{D}}\left(\left(\left(\mathbf{B} \bar{U}^{-1}\right) \cdot \bar{U}^{0}\right) \bar{h}_{1}^{0}+\frac{1}{2}\left(\left(\mathbf{B} \bar{U}^{-1}\right) \cdot \bar{U}^{-1}\right) \bar{h}_{1}^{1}\right) d t \\
\mathcal{E}^{1}\left(\tilde{u}_{ \pm}^{0}, \tilde{u}_{ \pm}^{1}, \bar{u}^{0}, \bar{u}^{1}, \bar{u}^{2}\right):= & \int_{\bar{D}_{ \pm}}\left(\mathbf{a}_{ \pm}\left(e\left(\tilde{u}_{ \pm}^{0}\right)\right) \cdot e\left(\tilde{u}_{ \pm}^{1}\right)-\tilde{f} \cdot \tilde{u}_{ \pm}^{1}\right) \tilde{h}_{1}^{0} d t-\int_{\tilde{I}_{g}}\left(\tilde{g} \cdot \tilde{u}_{ \pm}^{1}\right) \tilde{h}_{s}^{0} d \tilde{\alpha} \\
& +\int_{\bar{D}}\left(\left(\left(\mathbf{B} \bar{U}^{-1}\right) \cdot \bar{U}^{1}+\frac{1}{2}\left(\mathbf{B} \bar{U}^{0}\right) \cdot \bar{U}^{0}\right) \bar{h}_{1}^{0}\right) d t \\
& +\int_{\bar{D}}\left(\left(\left(\mathbf{B} \bar{U}^{-1}\right) \cdot \bar{U}^{0}\right) \bar{h}_{1}^{1}+\frac{1}{2}\left(\left(\mathbf{B} \bar{U}^{-1}\right) \cdot \bar{U}^{-1}\right) \bar{h}_{1}^{2}\right) d t \\
\mathcal{E}^{2}\left(\tilde{u}_{ \pm}^{0}, \tilde{u}_{ \pm}^{1}, \tilde{u}_{ \pm}^{2}, \bar{u}^{0}, \bar{u}^{1}, \bar{u}^{2}, \bar{u}^{3}\right):= & \int_{\bar{D}_{ \pm}}\left(\mathbf{a}_{ \pm}\left(e\left(\tilde{u}_{ \pm}^{0}\right)\right) \cdot e\left(\tilde{u}_{ \pm}^{2}\right)-\tilde{f} \cdot \tilde{u}_{ \pm}^{2}\right) \tilde{h}_{1}^{0} d t-\int_{\tilde{I}_{g}}\left(\tilde{g} \cdot \tilde{u}_{ \pm}^{2}\right) \tilde{h}_{s}^{0} d \tilde{\alpha} \\
& +\int_{\bar{D}_{ \pm}}\left(\frac{1}{2} \mathbf{a}_{ \pm}\left(e\left(\tilde{u}_{ \pm}^{1}\right)\right) \cdot e\left(\tilde{u}_{ \pm}^{1}\right)\right) \tilde{h}_{1}^{0} d t \\
& +\int_{\bar{D}}\left(\left(\left(\mathbf{B} \bar{U}^{-1}\right) \cdot \bar{U}^{2}+\left(\mathbf{B} \bar{U}^{0}\right) \cdot \bar{U}^{1}\right) \bar{h}_{1}^{0}\right) d t \\
& +\int_{\bar{D}}\left(\left(\left(\mathbf{B} \bar{U}^{-1}\right) \cdot \bar{U}^{1}+\frac{1}{2}\left(\mathbf{B} \bar{U}^{0}\right) \cdot \bar{U}^{0}\right) \bar{h}_{1}^{1}\right) d t \\
& \left.+\int_{\bar{D}}\left(\left(\mathbf{B} \bar{U}^{-1}\right) \cdot \bar{U}^{0}\right) \bar{h}_{1}^{2}+\frac{1}{2}\left(\left(\mathbf{B} \bar{U}^{-1}\right) \cdot \bar{U}^{-1}\right) \bar{h}_{1}^{3}\right) d t
\end{aligned}
$$

In these relations, the functions $\bar{U}^{l}, l=0,1,2, \ldots$, are the terms of the expansion

$$
\bar{U}^{\varepsilon}=\bar{U}^{0}+\varepsilon \bar{U}^{1}+\varepsilon^{2} \bar{U}^{2}+o\left(\varepsilon^{2}\right)
$$


and they are given by the relations:

$$
\begin{aligned}
\bar{U}^{-1}= & \left(\begin{array}{c}
0 \\
\bar{u}_{, 2}^{0}
\end{array}\right), \\
\bar{U}^{0}= & \left(\begin{array}{c}
\bar{u}_{, 1}^{0}+\frac{\bar{h}_{1,2}^{1}}{\bar{h}_{1}^{0}} \bar{v}^{0} \\
\bar{u}_{, 2}^{1}
\end{array}\right), \\
\bar{U}^{1}= & \left(\begin{array}{c}
\left(\frac{\bar{u}_{1,1}^{1}}{\bar{h}_{1}^{0}}+\frac{\bar{h}_{1,2}^{1}}{\bar{h}_{1}^{0}} \bar{v}^{1}\right)-\bar{u}_{, 1}^{0} \frac{\bar{h}_{1}^{1}}{\left(\bar{h}_{1}^{0}\right)^{2}}+\frac{\left(\bar{h}_{1,2}^{2} \bar{h}_{1}^{0}-\bar{h}_{1,2}^{1} \bar{h}_{1}^{1}\right)}{\left(\bar{h}_{1}^{0}\right)^{2}} \bar{v}^{0} \\
\bar{u}_{, 2}^{2}
\end{array}\right), \\
\bar{U}^{2}= & \left(\begin{array}{c}
\left(\frac{\bar{u}_{, 1}^{2}}{\bar{h}_{1}^{0}}+\frac{\bar{h}_{1,2}^{1}}{\bar{h}_{1}^{0}} \bar{v}^{2}\right)+\bar{u}_{, 1}^{0} \frac{\left(\left(\bar{h}_{1}^{1}\right)^{2}-\bar{h}_{1}^{2} \bar{h}_{1}^{0}\right)}{\left(\bar{h}_{1}^{0}\right)^{3}}+\frac{\left(\bar{h}_{1,2}^{2} \bar{h}_{1}^{0}-\bar{h}_{1,2}^{1} \bar{h}_{1}^{1}\right)}{\left(\bar{h}_{1}^{0}\right)^{2}} \bar{v}^{1} \\
\bar{u}_{, 2}^{3}
\end{array}\right) \\
& +\left(\begin{array}{c}
\left(\frac{\bar{h}_{1,2}^{1}}{\left(\bar{h}_{1}^{0}\right)^{3}}\left(\left(\bar{h}_{1}^{1}\right)^{2}-\bar{h}_{1}^{2} \bar{h}_{1}^{0}\right)-\frac{\bar{h}_{1,2}^{2}}{\left(\bar{h}_{1}^{0}\right)^{2}} \bar{h}_{1}^{1}+\frac{\bar{h}_{1,2}^{3}}{\left.\bar{h}_{1}^{0}\right) \bar{v}^{0}}\right. \\
0
\end{array}\right) .
\end{aligned}
$$

We note that the terms $\bar{U}^{l}, l=1,2, \ldots$, do not enter the energy $\mathcal{E}^{-1}$ because the zero order term of $\bar{h}^{\varepsilon} 1,2\left(\bar{h}^{\varepsilon} 1\right)^{-1}$ vanishes.

The last step of the asymptotic method is based on the following main assumption: fields which are stationary points of the energy $\mathcal{E}^{\varepsilon}$ can be obtained by finding the stationary points of the energies $\mathcal{E}^{l}, l=-1,0,1,2, \ldots$ The analysis of the stationary points of the energies at the various levels allows to identify the conditions between $\bar{u}^{0}, \bar{u}^{1}, \bar{u}^{2}, \ldots$ and the corresponding stress vectors arising from $\tilde{u}_{ \pm}^{0}, \tilde{u}_{ \pm}^{1}, \tilde{u}_{ \pm}^{2}, \ldots$ at the interfaces $S_{+}, S_{-}$.

\subsection{Stationary points of $\mathcal{E}^{-1}$}

In view of (38), the energy $\mathcal{E}^{-1}$ reduces to

$$
\mathcal{E}^{-1}\left(\bar{u}^{0}\right)=\int_{\bar{D}} \frac{1}{2}\left(\left(\mathbf{B}^{22} \bar{u}_{, 2}^{0}\right) \cdot \bar{u}_{, 2}^{0}\right) \bar{h}_{1}^{0} d t
$$

to be minimized in the class of displacements $\bar{u}^{0} \in H\left(\bar{B} ; R^{3}\right)$. Since $\mathbf{b}$ is a positive definite tensor, the matrix $\mathbf{B}^{22}$ is also positive definite. Therefore, the energy $\mathcal{E}^{-1}$ is non negative and the minimizers have the property that

$$
\bar{u}_{, 2}^{0}=0, \text { a.e. in } \bar{B},
$$

i. e. the minimizing displacements are independent of $t_{2}$ in the interphase. Based on this result and on the initial assumption of perfect contact between the interphase and the adherents, we obtain the vanishing of the jump of $\tilde{u}^{0}$ across $\bar{B}$ :

$$
\left[\tilde{u}^{0}\right]=0
$$




\subsection{Stationary points of $\mathcal{E}^{0}$}

From (43) it follows that $\bar{U}^{-1}=0$, and thus the energy $\mathcal{E}^{0}$ is independent of $\bar{u}^{0}, \bar{u}^{1}$. With a little abuse of notation, we drop the dependence of the these vector fields from the argument of $\mathcal{E}^{0}$, which becomes

$$
\mathcal{E}^{0}\left(\tilde{u}_{ \pm}^{0}\right):=\int_{\bar{D}_{ \pm}}\left(\frac{1}{2} \mathbf{a}_{ \pm}\left(e\left(\tilde{u}_{ \pm}^{0}\right)\right) \cdot e\left(\tilde{u}_{ \pm}^{0}\right)-\tilde{f} \cdot \tilde{u}_{ \pm}^{0}\right) \tilde{h}_{1}^{0} d t-\int_{\tilde{I}_{g}}\left(\tilde{g} \cdot \tilde{u}_{ \pm}^{0}\right) \tilde{h}_{s}^{0} d \tilde{\alpha}
$$

We seek the energy minimizer in the class of displacements

$$
V=\left\{\left(\tilde{u}_{ \pm}\right) \in H\left(\Omega_{ \pm} ; R^{3}\right): \tilde{u}_{+}\left(t_{1},+\frac{1}{2}\right)=\tilde{u}_{-}\left(t_{1},-\frac{1}{2}\right), t_{1} \in(a, b), \quad \tilde{u}_{ \pm}=0 \text { on } \tilde{\Gamma}_{u}\right\} .
$$

Using standard arguments, we obtain the equilibrium equations

$$
\begin{aligned}
& \operatorname{div}\left(\mathbf{a}_{ \pm}\left(e\left(\tilde{u}_{ \pm}^{0}\right)\right)+\tilde{f}\right)=0 \quad \text { in } \tilde{\Omega}_{ \pm}, \\
& \mathbf{a}_{ \pm}\left(e\left(\tilde{u}_{ \pm}^{0}\right)\right) n=\tilde{g} \text { on } \tilde{\Gamma_{g}}, \\
& \mathbf{a}_{ \pm}\left(e\left(\tilde{u}_{ \pm}^{0}\right)\right) n=0 \quad \text { on } \partial \tilde{\Omega}_{ \pm} \backslash \tilde{\Gamma}_{g},
\end{aligned}
$$

Denoted $\tilde{\sigma}^{0} n$ the traction vector, we also find that its jump across the rescaled curved interphase $\bar{B}$ vanishes:

$$
\left[\tilde{\sigma}^{0} n\right]=0 .
$$

From the mechanical viewpoint, condition (44) and (50) give a perfect interface condition for the interphase modeling.

\subsection{Stationary points of $\mathcal{E}^{1}$}

The vanishing of $\bar{U}^{-1}$ makes $\mathcal{E}^{1}$ independent of $\bar{u}^{2}$. Again with a little abuse of notation, we drop the dependence of this vector field in the argument of $\mathcal{E}^{1}$, which simplifies as

$$
\begin{aligned}
\mathcal{E}^{1}\left(\tilde{u}_{ \pm}^{0}, \tilde{u}_{ \pm}^{1}, \bar{u}^{0}, \bar{u}^{1}\right):= & \int_{\bar{D}_{ \pm}}\left(\mathbf{a}_{ \pm}\left(e\left(\tilde{u}_{ \pm}^{0}\right)\right) \cdot e\left(\tilde{u}_{ \pm}^{1}\right)-\tilde{f} \cdot \tilde{u}_{ \pm}^{1}\right) \tilde{h}_{1}^{0} d t-\int_{\tilde{I}_{g}}\left(\tilde{g} \cdot \tilde{u}_{ \pm}^{1}\right) \tilde{h}_{s}^{0} d \tilde{\alpha} \\
& +\int_{\bar{D}}\left(\frac{1}{2}\left(\mathbf{B} \bar{U}^{0}\right) \cdot \bar{U}^{0}\right) \bar{h}_{1}^{0} d t
\end{aligned}
$$

In view of equilibrium equations $(47 \div 49)$, the Euler-Lagrange equations of $\mathcal{E}^{1}$ take the form

$$
\tilde{\sigma}^{0} n=\mathbf{B}^{22}\left(\bar{u}_{, 2}^{1}\right)+\mathbf{B}^{21}\left(\frac{\bar{u}_{, 1}^{0}}{\bar{h}_{1}^{0}}+\frac{\bar{h}_{1,2}^{1}}{\bar{h}_{1}^{0}} \bar{v}^{0}\right) .
$$

This relation together with the continuity condition (44) gives the following condition on the jump in the displacement vector field $\bar{u}^{1}$ across the interphase

$$
\left[\bar{u}^{1}\right]=\left(\mathbf{B}^{22}\right)^{-1}\left(\tilde{\sigma}^{0} n-\mathbf{B}^{21}\left(\frac{\bar{u}_{, 1}^{0}}{\bar{h}_{1}^{0}}+\frac{\bar{h}_{1,2}^{1}}{\bar{h}_{1}^{0}} \bar{v}^{0}\right)\right) .
$$


Note that in view of the continuity of the displacement fields at the interfaces $S^{ \pm}$, the latter condition can be rewritten in the equivalent form

$$
\left[\tilde{u}^{1}\right]=\left(\mathbf{B}^{22}\right)^{-1}\left(\tilde{\sigma}^{0} n-\mathbf{B}^{21}\left(\frac{\bar{u}_{, 1}^{0}}{\bar{h}_{1}^{0}}+\frac{\bar{h}_{1,2}^{1}}{\bar{h}_{1}^{0}} \bar{v}^{0}\right)\right) .
$$

\subsection{Stationary points of $\mathcal{E}^{2}$}

Using the divergence theorem, equation (43), the equilibrium equations $(47 \div$ 49), and the jump conditions (54), we eliminate $\tilde{u}_{ \pm}^{0}, \tilde{u}_{ \pm}^{2}$ and $\bar{u}^{3}$ from the expression for the energy $\mathcal{E}^{2}$ and we simplify this expression as follows:

$$
\begin{aligned}
\mathcal{E}^{2}\left(\tilde{u}_{ \pm}^{0}, \tilde{u}_{ \pm}^{1}, \bar{u}^{0}, \bar{u}^{1}\right):= & \int_{\bar{D}_{ \pm}}\left(\frac{1}{2} \mathbf{a}_{ \pm}\left(e\left(\tilde{u}_{ \pm}^{1}\right)\right) \cdot e\left(\tilde{u}_{ \pm}^{1}\right)\right) \tilde{h}_{1}^{0} d t \\
& +\int_{\bar{D}}\left(\left(\mathbf{B}^{11} \bar{U}_{1}^{0}+\mathbf{B}^{12}\left(\mathbf{B}^{22}\right)^{-1}\left(\tilde{\sigma}^{0} n-\mathbf{B}^{21} \bar{U}_{1}^{0}\right)\right) \cdot \bar{U}_{1}^{1}\right) \bar{h}_{1}^{0} d t
\end{aligned}
$$

where $\bar{U}_{1}^{0}$ and $\bar{U}_{1}^{1}$ are the first components of the vectors $\bar{U}^{0}$ and $\bar{U}^{1}$, respectively (see $(39,40)$ ). In view of equation $(52)$ and noting that the continuity of the displacement vector field implies that $\tilde{u}_{ \pm}^{1}=\bar{u}^{1}$ at $S_{ \pm}$, the vector field $\bar{u}^{1}$ can be written in the following form:

$$
\bar{u}^{1}\left(t_{1}, t_{2}\right)=\left[\bar{u}^{1}\right]\left(t_{1}\right) t_{2}+\frac{1}{2} S\left(\tilde{u}^{1}\right)\left(t_{1}\right)
$$

where $S\left(\tilde{u}^{1}\right)\left(t_{1}\right):=\tilde{u}^{1}\left(t_{1}, 1 / 2\right)+\tilde{u}^{1}\left(t_{1},-1 / 2\right)$. Substituting $(14,54,56)$ into $(55)$ and integrating with respect to $t_{2}$ (note that $\bar{h}_{1}^{0}$ is independent of $t_{2}$ and $\bar{h}_{1}^{1}$ depends linearly upon $t_{2}$ ) further simplifies the energy as follows:

$$
\begin{aligned}
\mathcal{E}^{2}\left(\tilde{u}_{ \pm}^{0}, \tilde{u}_{ \pm}^{1}, \bar{u}^{0}, \bar{u}^{1}\right):= & \int_{\bar{D}_{ \pm}}\left(\frac{1}{2} \mathbf{a}_{ \pm}\left(e\left(\tilde{u}_{ \pm}^{1}\right)\right) \cdot e\left(\tilde{u}_{ \pm}^{1}\right)\right) \tilde{h}_{1}^{0} d t \\
& +\int_{\bar{D}}\left(F_{0} \cdot\left(\frac{1}{2}\left(S\left(\tilde{u}^{1}\right)\right)_{, 1}+\frac{\bar{h}_{1,2}^{1}}{2}\left(S\left(\tilde{u}^{1}\right) \wedge e_{3}\right)\right) \bar{h}_{1}^{0} d t\right.
\end{aligned}
$$

with

$$
F_{0}:=\left(\mathbf{B}^{11}-\mathbf{B}^{12}\left(\mathbf{B}^{22}\right)^{-1} \mathbf{B}^{21}\right) \bar{U}_{1}^{0}+\mathbf{B}^{12}\left(\mathbf{B}^{22}\right)^{-1} \tilde{\sigma}^{0} n
$$


The Euler-Lagrange equations for the minimization problem of the functional (57) are given by the relations

$$
\begin{aligned}
& \operatorname{div}\left(a_{ \pm}\left(e\left(\tilde{u}_{ \pm}^{1}\right)\right)\right)=0 \quad \text { in } \tilde{\Omega}_{ \pm} \\
& a_{ \pm}\left(e\left(\tilde{u}_{ \pm}^{1}\right)\right) n=0 \quad \text { on } \tilde{\Gamma}_{g} \\
& a_{ \pm}\left(e\left(\tilde{u}_{ \pm}^{1}\right)\right) n=0 \quad \text { on } \partial \tilde{\Omega}_{ \pm} \backslash \tilde{\Gamma}_{g} \\
& -a_{+}\left(e\left(\tilde{u}_{+}^{1}\right)\right) n-\frac{1}{2 \bar{h}_{0}^{1}} F_{0,1}-\frac{\bar{h}_{1,2}^{1}}{2 \bar{h}_{0}^{1}}\left(F_{0} \wedge e_{3}\right)=0 \quad \text { on }(a, b) \times\left\{+\frac{1}{2}\right\} \\
& a_{-}\left(e\left(\tilde{u}_{-}^{1}\right)\right) n-\frac{1}{2 \bar{h}_{0}^{1}} F_{0,1}-\frac{\bar{h}_{1,2}^{1}}{2 \bar{h}_{0}^{1}}\left(F_{0} \wedge e_{3}\right)=0 \quad \text { on }(a, b) \times\left\{-\frac{1}{2}\right\},
\end{aligned}
$$

plus conditions at the points $t_{1}=a, b$, enlightening the presence of concentrated forces at these points (see [1, equation (10)] ). We now add equations $(62,63)$ together to obtain the following relation for the jump in the traction at order one

$$
\left[\tilde{\sigma}^{1} n\right]=-\frac{1}{\bar{h}_{0}^{1}} F_{0,1}-\frac{\bar{h}_{1,2}^{1}}{\bar{h}_{0}^{1}}\left(F_{0} \wedge e_{3}\right) .
$$

Relations (54) and (64) are (nonlocal) laws for imperfect contact in the minimization problem associated with the rescaled energy (20).

\section{An example: circular isotropic interphase}

In this section we consider the case of a circular inclusion bounded by a thin adhesive layer of small thickness $\varepsilon$. In the rescaled domain, the adhesive occupies the region

$$
\bar{B}=\left\{x \in \mathbb{R}^{2}: x=\left(R+\varepsilon t_{2}\right) n\left(t_{1}\right), \quad\left(t_{1}, t_{2}\right) \in[0,2 \pi) \times(-1 / 2,1 / 2)\right\},
$$

where $n\left(t_{1}\right)$ denotes the unit normal to the boundary of the adhesive, see figure 2 . In the figure, $R^{+}:=R+1 / 2$ and $R^{-}:=R-1 / 2$ denote the outer and inner radii of the adhesive layer. The metric coefficients are

$$
\begin{aligned}
& h_{1}=R+\varepsilon t_{2}, \\
& h_{2}=1 .
\end{aligned}
$$

Assuming that the adhesive is composed of isotropic material with Lamé constants $\lambda, \mu$ and considering a system of polar coordinates $(r, \theta)$, we obtain that 


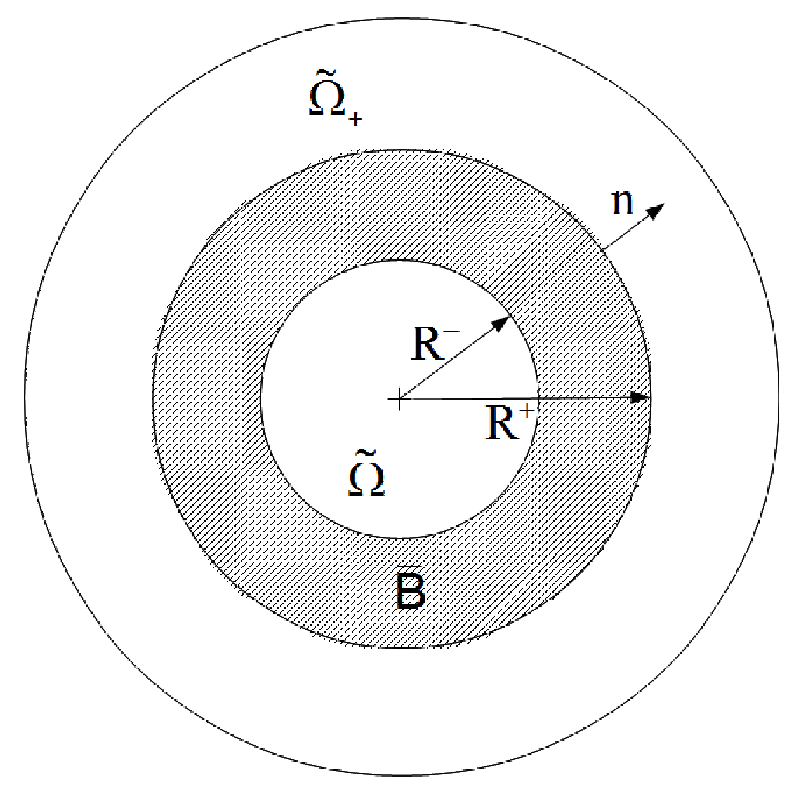

Figure 2: Rescaled configuration of a circular interphase $\bar{B}$ of unit thickness placed between two adherents.

relations (54) and (64) specialize as follows:

$$
\begin{aligned}
{\left[\tilde{u}_{\theta}^{1}\right] } & =\frac{1}{\mu} \sigma_{r \theta}^{0}-\frac{1}{R}\left(\tilde{u}_{\theta}^{0}-\tilde{u}_{r, \theta}^{0}\right), \\
{\left[\tilde{u}_{r}^{1}\right] } & =\frac{1}{(2 \mu+\lambda)} \sigma_{r r}^{0}-\frac{1}{R} \frac{\lambda}{(2 \mu+\lambda)}\left(\tilde{u}_{\theta, \theta}^{0}+\tilde{u}_{r}^{0}\right), \\
R\left[\tilde{\sigma}_{r \theta}^{1}\right] & =-\frac{4 \mu(\lambda+\mu)}{(2 \mu+\lambda)} \frac{1}{R}\left(\tilde{u}_{\theta, \theta \theta}^{0}+\tilde{u}_{r, \theta}^{0}\right)-\frac{\lambda}{(2 \mu+\lambda)} \sigma_{r r, \theta}^{0}-\sigma_{r \theta}^{0}, \\
R\left[\tilde{\sigma}_{r r}^{1}\right] & =\frac{4 \mu(\lambda+\mu)}{(2 \mu+\lambda)} \frac{1}{R}\left(\tilde{u}_{\theta, \theta}^{0}+\tilde{u}_{r}^{0}\right)+\frac{\lambda}{(2 \mu+\lambda)} \sigma_{r r}^{0}-\sigma_{r \theta, \theta}^{0} .
\end{aligned}
$$

\section{Conclusion}

In this paper, we obtained the interface law mechanically equivalent to the effect of a curved thin elastic anisotropic adhesive interphase undergoing plane deformations. The derivation of the interface law was based on an energy approach proposed in [14] and based on two main assumptions: the possible existence of expansions in series in terms of the interphase thickness of the displacement and stress fields, and the assumption that the fields which are stationary points of the energy of the system can be obtained by finding the stationary points of the energies obtained at each level in the expansion. The main result is that at the order zero the interphase behaves as an ideal interface across which the displacements and tractions are continuous (see (44) and (50)). At the next order the 
interphase is modeled by an imperfect interface, whose contact law prescribes the jumps of the displacements and of the traction vector fields at order one in terms of the corresponding fields obtained at the order zero (see (54) and (64)). As an example, the case of a linear elastic isotropic thin interphase bounding a circular inclusion was considered and the equivalent imperfect interface law was obtained.

Acknowledgement. RR acknowledges the support from the Italian Ministry of University and Research, Project PRIN 2009 "Modellazione multi-scala di materiali e strutture", coordinated by A. Corigliano.

\section{References}

[1] R. Abdelmoula and M. Coutris and J. Marigo, "Comportment asymptotique d'une interphase élasticque mince", Compte Rendu Académie des Sciences Série IIb, 326, 237-242, 1998.

[2] Y. Benveniste, "A general interface model for a three-dimensional curved thin anisotropic interphase between two anisotropic media", Journal of the Mechanics and Physics of Solids 54, 708-734, 2006.

[3] Y. Benveniste and T. Miloh, "Imperfect soft and stiff interfaces in twodimensional elasticity", Mechanics of Materials, 33, 309-324, 2001.

[4] D. Caillerie, "The effect of a thin inclusion of high rigidity in an elastic body", Mathematical Methods in Applied Sciences, 2, 251-270, 1980.

[5] P. G. Ciarlet, "Mathematical Elasticity. Volume I: Three-Dimensional Elasticity", North-Holland, Amsterdam, 1988.

[6] J.F. Ganghoffer and A. Brillard and J. Schultz, "Modelling of the mechanical behaviour of joints bonded by a nonlinear incompressible elastic adhesive", European Journal of Mechanics A/Solids, 16, 255-276, 1997.

[7] G. Geymonat and F. Krasucki and S. Lenci, "Mathematical Analysis of a bonded joint with a soft thin adhesive", Mathematics and Mechanics of Solids, 16, 201-225, 1999.

[8] G. Geymonat and F. Krasucki, "Analyse asymptotique du comportement en flexion de deux plaques collées", Compte Rendu Académie des Sciences Série I, 325, 307-314, 1997.

[9] Z. Hashin, "Thin interphase/imperfect interface in elasticity with application to coated fiber composites", Journal of the Mechanics and Physics of Solids, 50 (12), 2002, 2509-2537.

[10] A. Klarbring, "Derivation of the adhesively bonded joints by the asymptotic expansion method", International Journal of Engineering Science, 29, 493512, 1991. 
[11] A. Klarbring, A.B. Movchan, "Asymptotic modeling of adhesive joints", Mechanics of Materials, 28, 137-145, 1998.

[12] F. Lebon and R. Rizzoni, "Asymptotic study of soft thin layer: the non convex case", Mechanics of Advanced Materials and Structures, 15, 12-20, 2008.

[13] F. Lebon and R. Rizzoni, "Asymptotic analysis of a thin interface: the case involving similar rigidity", International Journal of Engineering Sciences, 48, 473-486, 2010.

[14] F. Lebon and R. Rizzoni, "Asymptotic behavior of a hard thin linear elastic interphase: an energy approach", International Journal of Solids and Structures, 48, 441-449, 2011.

[15] F. Lebon and R. Rizzoni, "Modelling Adhesion by Asymptotic Techniques", in "Adhesive properties in nanomaterials, composites and films", Nova Publishers, 95-126, 2011.

[16] F. Lebon and R. Rizzoni, "Asymptotic analysis of an adhesive joint with mismatch strain", European Journal of Mechanics - A/Solids, in press, 2012.

[17] F. Lebon and R. Rizzoni and S. Ronel, "Analysis of non-linear soft thin interfaces", Computers and Structures, 82, 1929-1938, 2004.

[18] F. Lebon and S. Ronel-Idrissi, "Asymptotic analysis of Mohr-Coulomb and Drucker-Prager soft thin layers", International Journal of Steel and Composite Structures, 4, 133-148, 2004.

[19] F. Lebon and S. Ronel, "First order numerical analysis of linear thin layers, ASME Journal of Applied Mechanics", 74, 824-828, 2007.

[20] F. Lebon and A. Ould-Khaoua and C. Licht, "Numerical study of soft adhesively bonded joints in finite elasticity", Computational Mechanics, 21, 134-140, 1997.

[21] F. Lebon and F. Zaittouni, "Asymptotic modelling of interface taking into account contact conditions: Asymptotic expansions and numerical implementation", International Journal of Engineering Sciences, 48, 111-127, 2010 .

[22] C. Licht and G. Michaille, "A modeling of elastic adhesive bonded joints", Advances in Mathematical Sciences and Applications, 7, 711-740, 1997.

[23] C. Pelissou and F. Lebon, "Asymptotic modeling of quasi-brittle interfaces", Computers and Structures, 87, 1216-1223, 2009.

[24] M. Serpilli, S. Lenci, "Limit models in the analysis of three different layered elastic strips", European Journal of Mechanics - A/Solids, 27 (2), 247-268, 2008.

[25] F. Zaittouni and F. Lebon and C. Licht, "Etude théorique et numérique du comportement d'un assemblage de plaques", Compte Rendu Académie des Sciences Série Mécanique, 330, 359-364, 2002. 